\title{
Patterns of Femoral Bone Remodeling: Comparison of the Tongariki Native Easter Islanders with European Population
}

\author{
Andrea G. Drusini*, ${ }^{,}$, Annunziata Carrieri ${ }^{1}$ and Anna Chiara Frigo ${ }^{2}$ \\ ${ }^{I}$ Department of Medical-Diagnostic Sciences and Special Therapies, University of Padova, Via Giustiniani 2- 35128 \\ Padova, Italy \\ ${ }^{2}$ Dipartimento di Medicina Ambientale e Sanità Pubblica - Sede di Igiene, Università di Padova, Via Loredan 18, 35131 \\ Padova, Italy
}

\begin{abstract}
The relationship between sex, age and histomorphometry in femoral cortical bone was examined in a $19^{\text {th }}$ century skeletal population from the XIX-XX Century Northern Italy, and compared with the femoral sections belonging to the Tongariki Easter Islanders (XVII-XVIII Century A.D.). Femoral cross sections were examined using an image analysis system. Several histological variables were calculated to assess differences between sexes and among age groups. The results indicate significant differences between the two populations in osteon remodeling. OPD (Osteon Population Density) increased especially in the female sample in both populations, but In the Easter Island female sample OA (Osteon Area) was lower than in the European female population. In the Easter Island female sample, \%Or (percent Osteon refilling) decreased after 49 years of age. The phenomenon was also evident in both sexes of European population, although the involution process was not so marked as age advanced.
\end{abstract}

Keywords: Easter Island, osteon remodeling, moai building, stress.

\section{INTRODUCTION}

Rapa Nui is a hilly, high volcanic island of 64 square miles, settled by Polynesians derived from Asia and spoke an eastern Polynesian dialect related to Hawaiian and Marquesan. The giant statues of Easter Island - the moai - range from 2 to almost $10 \mathrm{~m}$ in height, and weight can reach the 82 tons. Archaeological evidence confirms intense building activity - especially moai building - on the island for 500 years. Hundreds of statues were moved from the quarry, some of them as far as $10 \mathrm{~km}\left(6 \frac{1}{4}\right.$ miles). Since 180 people could pull a 10 tons statue, as an experiment demonstrated, specialists in ancient technology calculate that 1500 people could certainly have moved a 82 tons statue. Anthropological studies revealed that humeral and femoral robusticity indices of Easter islanders were very similar in males and females; it can be supposed that also women were submitted to intense physical stresses [1]. Easter Island (Rapa Nui) is currently the focal point of research in an intensively studied archaeological area that extends from the plains at the foot of the southwest slopes of the Poike Peninsula. Close to three hundred ceremonial or burial structures are known and recognized as ahu (ceremonial centre) on the whole island: of these, a smaller number are large, complex, raised, rectangular platforms, sloping ramps and associated 'plazas', most of which supporting multi-ton monolithic statues of volcanic tuff. The 'moai', symbols of the prestige and status of the local groups, raised to worship deified ancestors, are the most conspicuous features of the archaeological landscape. The analysis of radiocarbon-dated structures indicates that

*Address correspondence to this author at the Department of MedicalDiagnostic Sciences and Special Therapies, University of Padova, Via Giustiniani 2- 35128 Padova, Italy; Tel: 049-8276302; Fax: 049-8276300; E-mail: andrea.drusini@unipd.it the ahu and the statues represent a chronological progression of a single developing society [2].

During the period 1991-2001, the Italian Archaeological Mission directed by Giuseppe Orefici carried out some excavations in different sites of the island, and a huge quantity of skeletal remains belonging to pre-contact Easter islanders was recovered from the Ahu Tongariki ceremonial centre in an area of some 5000 square meters [1, 3]. All the bones were studied according to the standard physical anthropological methods, including sex and age determination, osteometrics, morphological variations, and palaeopathology. Moreover, histological sections were prepared from samples taken from femoral midshaft.

Bone possesses the unique property of providing a living, dynamic and durable record of past events encoded in its microstructure [4-7]. Quantitative histological studies of ancient bone can provide information relating to age at death and levels of physical activity [8-31]. Studies as far back as 1892 by Wolff have shown that bone is negatively influenced by reduction of its load-carrying role. Bone loss and reduction of functional-mechanical integrity of the tissue during life is mainly due to a decreased mechanical usage of skeleton. Mechanical stress is an important factor in the maintenance of normal cortical bone remodeling. Studies as well as bone density and histomorphometric analyses has been conducted in vivo to detect long-term bone loss resulting from long-term disuse, but few information was obtained from historical and archaeological skeletal material. As Burr et al. [26] have demonstrated, the data suggest that a more active lifestyle is associated with greater osteon population density (OPD). The aim of this study was a quantitative estimation of variation in bone microstructures in two populations, differing not only in geographic position, but also in 
social-economic and adaptive background. Histomorphometric analysis of femoral cortical bone, based on image analysis techniques, has recently become an important investigative method in the study of the histological variables of bone remodeling. The aim of this study was a quantitative estimation of variation in bone microstructures in two populations, differing not only in geographic position, but also in socialeconomic and adaptive background.

\section{MATERIALS AND METHODS}

Histological and histomorphometric analysis of femoral diaphyseal fragments (excluding the linea aspera, see Fig. 1) from twenty precontact native Easter Islanders $(9$ males and 11 females) were compared with those of 151 Europeans from the $19^{\text {th }}$ century (81 males and 70 females) of known age, sex, occupation and cause of death. Thin undecalcified histological sections of approximately 90 microns embedded in ethylmethacrylate were obtained using a Leitz 1600 rotating microtome. The sections were examined under low power with $10 \times$ ocular and $10 \times$ widefield ocular lenses using a standard laboratory microscope with a grid of $0.64 \mathrm{~mm}^{2}$ on the eyepiece. The following twelve histomorphometric variables were studied [7, 14, 26, 30, 32-34] (Tables 1 and 2):

- $\quad$ Intact Osteon Density (IO) - number of secondary intact osteons $\left(\mathrm{n}_{\mathrm{o}}\right)$ per $\mathrm{mm}^{2}$; secondary intact osteon is one where Haversian canal did not undergo remodeling at least for $90 \%$;

- $\quad$ Fragmentary Osteon Density (FR) - number of fragmentary osteons $\left(\mathrm{n}_{\mathrm{f}}\right)$ per $\mathrm{mm}^{2}$; fragmentary osteon is one where Haversian canal underwent remodeling at least for $10 \%$;

- $\quad$ Osteon Area $(\mathrm{OA})$ - area average of secondary osteon surfaces $\left(\mathrm{A}_{\mathrm{o}}\right)$, Haversian canals included;

- $\quad$ Osteon Perimeter $\left(\mathrm{P}_{\mathrm{o}}\right)$ - perimeter average of secondary osteon surfaces $\left(\mathrm{p}_{\mathrm{o}}\right)$, Haversian canals included;

- Haversian Canal Area (HcA) - area average of Haversian canal surfaces $\left(\mathrm{A}_{\mathrm{Hc}}\right)$;

- $\quad$ Haversian Canal Perimeter $\left(\mathrm{P}_{\mathrm{Hc}}\right)$ - perimeter average of Haversian canals $\left(\mathrm{p}_{\mathrm{Hc}}\right)$;

- $\quad$ Mean Osteonal Cross Sectional Area $\left(\mathrm{A}_{\mathrm{h}}\right)$ - area average of secondary osteon surfaces $\left(\mathrm{p}_{\mathrm{o}}\right)$, Haversian canals excluded

$\mathrm{A}_{\mathrm{h}}=\mathrm{OA}-\mathrm{HcA}$

- $\quad$ Mean Cross Sectional Diameter $\left(\mathrm{D}_{\mathrm{h}}\right)$ - diameter average of secondary osteon

$\mathrm{D}_{\mathrm{h}}=\sqrt{\frac{4 \mathrm{~A}_{\mathrm{h}}}{\pi}}$

- $\quad$ Osteon Population Density (OPD) - total number of intact secondary osteon $\left(\mathrm{n}_{\mathrm{o}}\right)$ per $\mathrm{mm}^{2}$ and total number of osteon fragments $\left(\mathrm{n}_{\mathrm{f}}\right)$ per $\mathrm{mm}^{2}$

$\mathrm{OPD}=\mathrm{IO}+\mathrm{FR}$

- $\quad$ Accumulated Osteon Creation (AOC) - total number of intact secondary osteons $\left(\mathrm{n}_{\mathrm{o}}\right)$ per $\mathrm{mm}^{2}$, total number of osteon fragments $\left(\mathrm{n}_{\mathrm{f}}\right)$ per $\mathrm{mm}^{2}$ and missing osteons (old osteons completely replaced by new ones and so not visible), for a specific OPD
$\mathrm{AOC}=\mathrm{IO}+\mathrm{FR}+$ Missing $_{\text {osteons }}$

Missing osteons were calculated following the methods proposed by Frost [32], Stout and Paine [34] and Abbott et al. [5].

- $\quad$ Net Osteonal Remodeling (nOR) - total quantification of bone remodeling

$\mathrm{nOR}=\mathrm{AOC} \times \mathrm{A}_{\mathrm{h}}$

nOR is a very important index independent of both chronological age and real compact bone age.

- $\quad$ Percent Osteonal Refilling (\%Or) - total quantification of bone refilling

$\% \mathrm{Or}=\frac{\mathrm{Ah}_{\mathrm{h}}}{\mathrm{OR}}$

The formula expresses the osteblast replacement modality due to the osteoclasts.

The two-tailed $t$ test was utilized in order to evaluate the significant differences among the variables within the age classes and between the two populations (Tables $\mathbf{3}, \mathbf{4}$ and $\mathbf{5}$ ). The sexual dimorphism index after Hall [35] was also used to quantify the differences between sexes (Table 6).

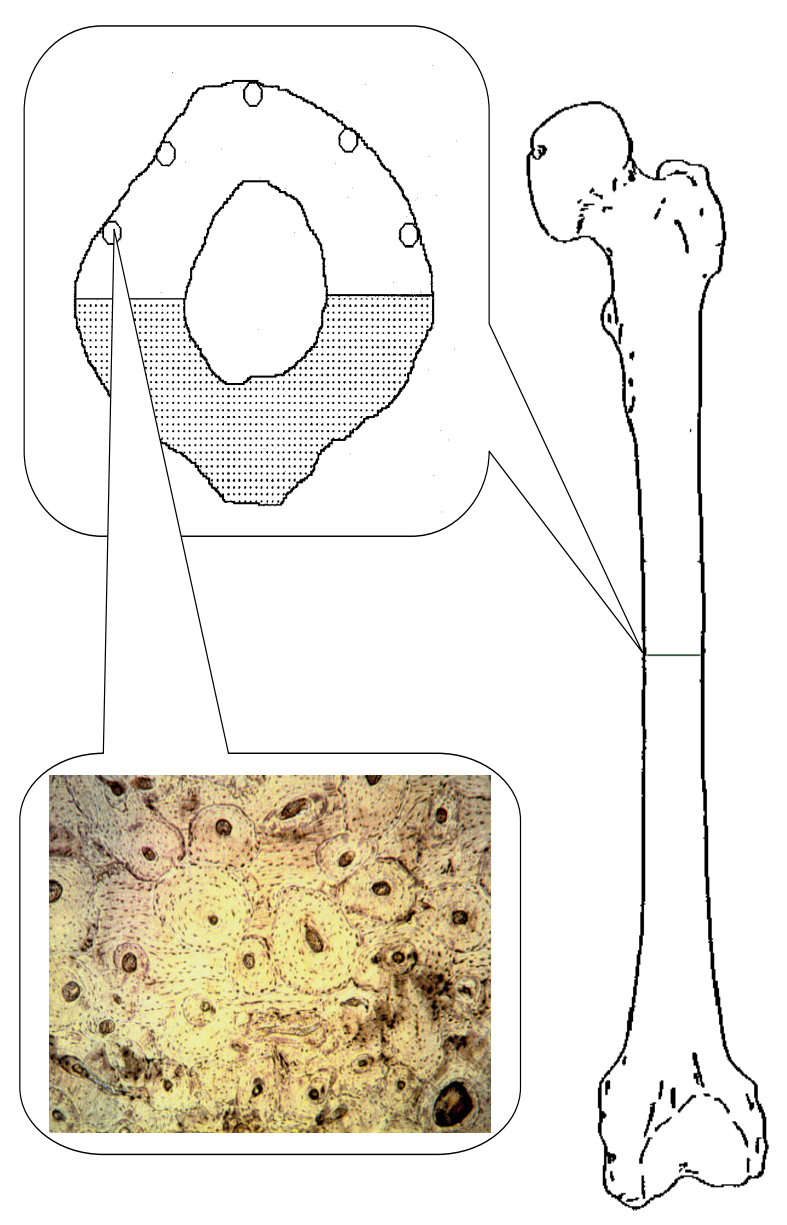

Fig. (1). Location of transverse section along the femural diaphysis. 


\section{RESULTS}

Table 1. Summary of the 12 Variables Considered Per Age Class and Sex in the European Population

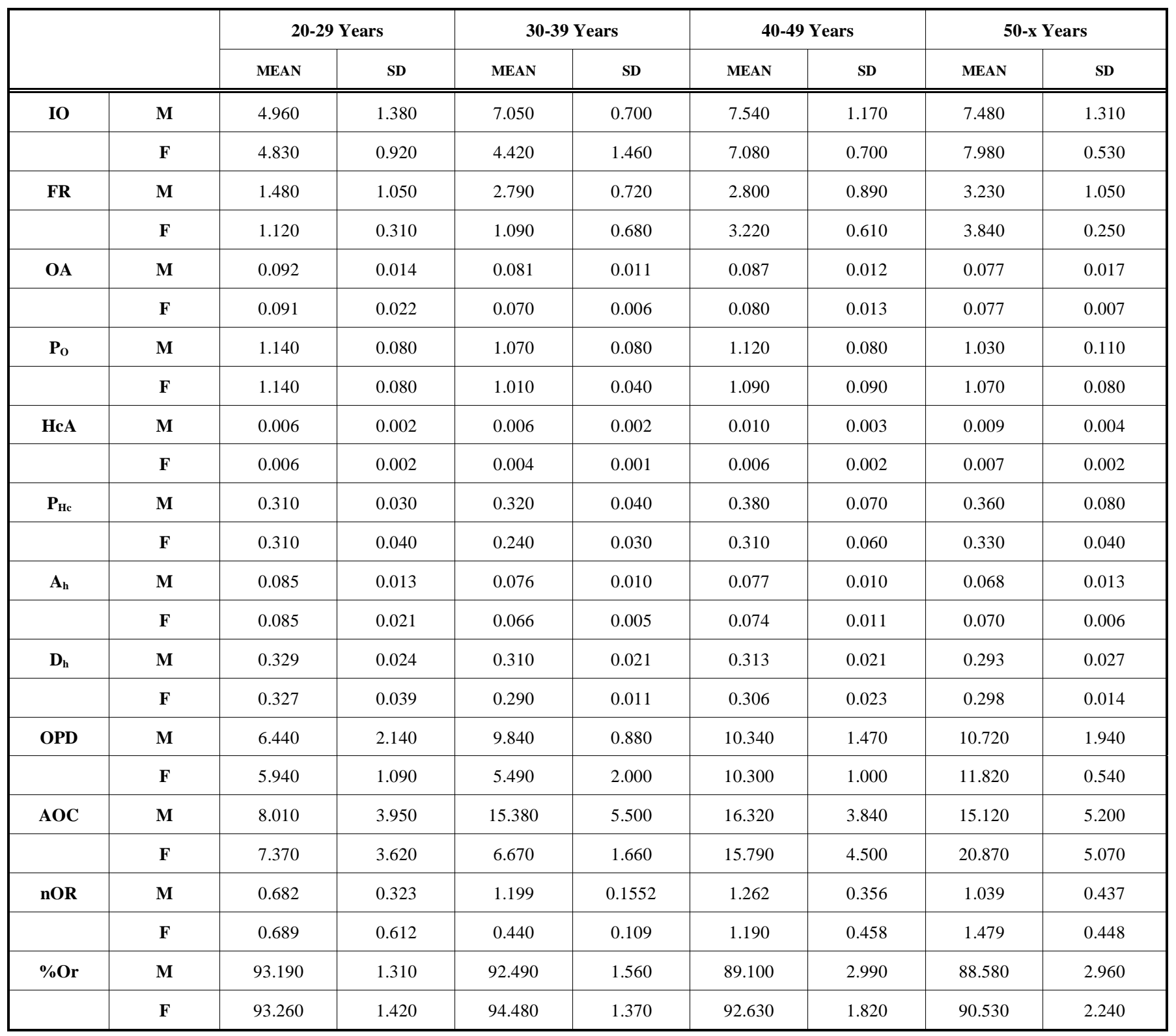

Table 2. Summary of the 12 Variables Considered Per Age Class and Sex in Easter Islanders

\begin{tabular}{|c|c|c|c|c|c|c|c|}
\hline & & \multicolumn{2}{|c|}{ 30-39 Years } & \multicolumn{2}{|c|}{ 40-49 Years } & \multicolumn{2}{|c|}{ 50-x Years } \\
\hline \multirow[t]{2}{*}{ IO } & $\mathbf{M}$ & - & - & 7.160 & 1.230 & 9.000 & 0.740 \\
\hline & $\mathbf{F}$ & 5.430 & 0.550 & 7.700 & 0.870 & 10.500 & 0.850 \\
\hline FR & $\mathbf{M}$ & - & - & 2.940 & 0.260 & 4.050 & 0.750 \\
\hline \multirow[t]{2}{*}{ OA } & $\mathbf{M}$ & - & - & 0.075 & 0.007 & 0.075 & 0.005 \\
\hline & $\mathbf{F}$ & 0.069 & 0.014 & 0.068 & 0.012 & 0.990 & 0.080 \\
\hline
\end{tabular}


(Table 2). Contd.....

\begin{tabular}{|c|c|c|c|c|c|c|c|}
\hline & & \multicolumn{2}{|c|}{ 30-39 Years } & \multicolumn{2}{|c|}{ 40-49 Years } & \multicolumn{2}{|c|}{ 50-x Years } \\
\hline & & MEAN & SD & MEAN & SD & MEAN & SD \\
\hline \multirow[t]{2}{*}{$\mathbf{P}_{\mathbf{O}}$} & $\mathbf{M}$ & - & - & 1.000 & 0.060 & 1.010 & 0.020 \\
\hline & $\mathbf{F}$ & 0.980 & 0.100 & 0.990 & 0.080 & 1.000 & 0.100 \\
\hline \multirow[t]{2}{*}{ HcA } & $\mathbf{M}$ & - & - & 0.009 & 0.003 & 0.009 & 0.002 \\
\hline & $\mathbf{F}$ & 0.008 & 0.002 & 0.008 & 0.002 & 0.015 & 0.008 \\
\hline \multirow[t]{2}{*}{$\mathbf{P}_{\mathrm{Hc}}$} & $\mathbf{M}$ & - & - & 0.390 & 0.060 & 0.370 & 0.030 \\
\hline & $\mathbf{F}$ & 0.360 & 0.040 & 0.370 & 0.060 & 0.440 & 0.090 \\
\hline \multirow[t]{2}{*}{$\mathbf{A}_{\mathbf{h}}$} & $\mathbf{M}$ & - & - & 0.066 & 0.005 & 0.067 & 0.004 \\
\hline & $\mathbf{F}$ & 0.061 & 0.012 & 0.059 & 0.010 & 0.055 & 0.007 \\
\hline \multirow[t]{2}{*}{$\mathbf{D}_{\mathrm{h}}$} & $\mathbf{M}$ & - & - & 0.289 & 0.011 & 0.291 & 0.010 \\
\hline & $\mathbf{F}$ & 0.278 & 0.028 & 0.274 & 0.023 & 0.264 & 0.016 \\
\hline \multirow[t]{2}{*}{ OPD } & $\mathbf{M}$ & - & - & 10.100 & 1.300 & 13.005 & 0.310 \\
\hline & $\mathbf{F}$ & 8.150 & 0.660 & 10.750 & 0.910 & 14.470 & 1.940 \\
\hline \multirow[t]{2}{*}{ AOC } & $\mathbf{M}$ & - & - & 13.540 & 4.610 & 26.800 & 7.690 \\
\hline & $\mathbf{F}$ & 8.850 & 0.910 & 12.950 & 1.420 & 24.820 & 8.470 \\
\hline \multirow[t]{2}{*}{ nOR } & $\mathbf{M}$ & - & - & 0.904 & 0.364 & 1.815 & 0.634 \\
\hline & $\mathbf{F}$ & 0.544 & 0.143 & 0.775 & 0.182 & 1.372 & 0.479 \\
\hline \multirow[t]{2}{*}{$\% \mathrm{Or}$} & $\mathbf{M}$ & - & - & 88.230 & 2.600 & 88.430 & 1.990 \\
\hline & $\mathbf{F}$ & 88.470 & 1.690 & 87.930 & 1.600 & 80.400 & 6.500 \\
\hline
\end{tabular}

Table 3.

AGE $=30-39$

The TTEST Procedure

Statistics

\begin{tabular}{|c|c|c|c|c|c|c|c|c|}
\hline $\begin{array}{l}\text { Variable } \\
\text { Wilcoxon }\end{array}$ & $\begin{array}{l}\text { Population Two- } \\
\text { Sample Test }\end{array}$ & $\mathbf{N}$ & Mean & Std Dev & Std Err & Minimum & Maximum & $\operatorname{Pr}>|t| \operatorname{Pr}$ \\
\hline IO & I & 15 & 6.3333 & 1.296 & 0.3346 & 3.8 & 8 & \\
\hline IO & $\mathrm{P}$ & 4 & 5.425 & 0.6305 & 0.3152 & 4.8 & 6.25 & \\
\hline IO & Diff & $(1-2)$ & 0.9083 & 1.2055 & 0.6784 & & 0.1982 & 0.1768 \\
\hline FR & I & 15 & 2.34 & 0.9898 & 0.2556 & 0.4 & 4 & \\
\hline FR & $\mathrm{P}$ & 4 & 2.725 & 0.3594 & 0.1797 & 2.4 & 3.2 & \\
\hline FR & Diff & $(1-2)$ & -0.385 & 0.9108 & 0.5126 & & 0.4628 & 0.4225 \\
\hline $\mathrm{OA}$ & I & 15 & 0.078 & 0.0112 & 0.0029 & 0.062 & 0.1 & \\
\hline $\mathrm{OA}$ & $\mathrm{P}$ & 4 & 0.0693 & 0.016 & 0.008 & 0.05 & 0.089 & \\
\hline $\mathrm{OA}$ & Diff & $(1-2)$ & 0.0087 & 0.0122 & 0.0069 & & 0.2188 & 0.4521 \\
\hline po & I & 15 & 1.054 & 0.0761 & 0.0197 & 0.95 & 1.22 & \\
\hline po & $\mathrm{P}$ & 4 & 0.9775 & 0.1109 & 0.0554 & 0.85 & 1.12 & \\
\hline po & Diff & $(1-2)$ & 0.0765 & 0.0833 & 0.0469 & & 0.1212 & 0.2920 \\
\hline $\mathrm{HcA}$ & I & 15 & 0.0055 & 0.0019 & 0.0005 & 0.003 & 0.009 & \\
\hline $\mathrm{HcA}$ & $\mathrm{P}$ & 4 & 0.0083 & 0.0026 & 0.0013 & 0.006 & 0.011 & \\
\hline
\end{tabular}


(Table 3). Contd.....

\begin{tabular}{|c|c|c|c|c|c|c|c|c|}
\hline $\begin{array}{l}\text { Variable } \\
\text { Wilcoxon }\end{array}$ & $\begin{array}{c}\text { Population Two- } \\
\text { Sample Test }\end{array}$ & $\mathbf{N}$ & Mean & Std Dev & Std Err & Minimum & Maximum & $\operatorname{Pr}>|t| \operatorname{Pr}$ \\
\hline $\mathrm{HcA}$ & Diff & $(1-2)$ & -0.003 & 0.0021 & 0.0012 & & 0.0319 & 0.0608 \\
\hline phe & I & 15 & 0.2967 & 0.0551 & 0.0142 & 0.21 & 0.39 & \\
\hline phc & $\mathrm{P}$ & 4 & 0.3575 & 0.0499 & 0.025 & 0.31 & 0.41 & \\
\hline phc & Diff & $(1-2)$ & -0.061 & 0.0542 & 0.0305 & & 0.0625 & 0.0796 \\
\hline $\mathrm{Ah}$ & I & 15 & 0.0726 & 0.01 & 0.0026 & 0.057 & 0.091 & \\
\hline $\mathrm{Ah}$ & $\mathrm{P}$ & 4 & 0.061 & 0.0139 & 0.0069 & 0.044 & 0.078 & \\
\hline $\mathrm{Ah}$ & Diff & $(1-2)$ & 0.0116 & 0.0108 & 0.0061 & & 0.0723 & 0.2696 \\
\hline $\mathrm{Dh}$ & I & 15 & 0.3035 & 0.0209 & 0.0054 & 0.27 & 0.34 & \\
\hline $\mathrm{Dh}$ & $\mathrm{P}$ & 4 & 0.2778 & 0.0323 & 0.0161 & 0.237 & 0.316 & \\
\hline $\mathrm{Dh}$ & Diff & $(1-2)$ & 0.0257 & 0.0233 & 0.0131 & & 0.0664 & 0.2933 \\
\hline OPD & I & 15 & 8.6733 & 2.0436 & 0.5277 & 4.2 & 11.4 & \\
\hline OPD & $\mathrm{P}$ & 4 & 8.15 & 0.7627 & 0.3813 & 7.2 & 9.05 & \\
\hline OPD & Diff & $(1-2)$ & 0.5233 & 1.882 & 1.0591 & & 0.6275 & 0.3951 \\
\hline $\mathrm{AOC}$ & I & 15 & 12.477 & 6.3719 & 1.6452 & 4.24 & 27.6 & \\
\hline AOC & $\mathrm{P}$ & 4 & 8.855 & 1.0514 & 0.5257 & 7.52 & 9.97 & \\
\hline AOC & Diff & $(1-2)$ & 3.6223 & 5.7993 & 3.2634 & & 0.2825 & 0.2501 \\
\hline nOR & I & 15 & 0.9457 & 0.5991 & 0.1547 & 0.291 & 2.512 & \\
\hline nOR & $\mathrm{P}$ & 4 & 0.5443 & 0.1642 & 0.0821 & 0.411 & 0.78 & \\
\hline nOR & Diff & $(1-2)$ & 0.4014 & 0.548 & 0.3084 & & 0.2104 & 0.2501 \\
\hline$\%$ Or & I & 15 & 93.157 & 1.8289 & 0.4722 & 90.35 & 95.78 & \\
\hline$\% \mathrm{Or}$ & $\mathrm{P}$ & 4 & 88.473 & 1.9481 & 0.9741 & 86.34 & 91.04 & \\
\hline$\% \mathrm{Or}$ & Diff & $(1-2)$ & 4.6842 & 1.8505 & 1.0413 & & 0.0003 & 0.0059 \\
\hline
\end{tabular}

Table 4.

$\mathrm{AGE}=40-49$

The TTEST Procedure

Statistics

\begin{tabular}{|c|c|c|c|c|c|c|c|c|}
\hline $\begin{array}{c}\text { Variable } \\
\text { PrWilcoxon }\end{array}$ & $\begin{array}{c}\text { Population Two- } \\
\text { Sample Test }\end{array}$ & $\mathbf{N}$ & Mean & Std Dev & Std Err & Minimum & Maximum & $\operatorname{Pr}>|t|$ \\
\hline IO & I & 14 & 7.2429 & 0.9573 & 0.2559 & 5.8 & 9.4 & \\
\hline IO & Diff & $(1-2)$ & -0.157 & 1.0502 & 0.4487 & & 0.7296 & 0.8747 \\
\hline FR & I & 14 & 3.0714 & 0.779 & 0.2082 & 1.2 & 4.6 & \\
\hline FR & Diff & $(1-2)$ & 0.0825 & 0.6316 & 0.2699 & & 0.7627 & 0.6350 \\
\hline $\mathrm{OA}$ & I & 14 & 0.0824 & 0.0131 & 0.0035 & 0.064 & 0.102 & \\
\hline $\mathrm{OA}$ & $\mathrm{P}$ & 9 & 0.0716 & 0.0106 & 0.0035 & 0.055 & 0.085 & \\
\hline $\mathrm{OA}$ & Diff & $(1-2)$ & 0.0109 & 0.0122 & 0.0052 & & 0.0494 & 0.0584 \\
\hline $\mathrm{HcA}$ & I & 14 & 0.0074 & 0.0032 & 0.0009 & 0.003 & 0.014 & \\
\hline
\end{tabular}


(Table 4). Contd.....

\begin{tabular}{|c|c|c|c|c|c|c|c|c|}
\hline $\begin{array}{c}\text { Variable } \\
\text { PrWilcoxon }\end{array}$ & $\begin{array}{c}\text { Population Two- } \\
\text { Sample Test }\end{array}$ & $\mathbf{N}$ & Mean & Std Dev & Std Err & Minimum & Maximum & $\operatorname{Pr}>|\mathbf{t}|$ \\
\hline $\mathrm{HcA}$ & $\mathrm{P}$ & 9 & 0.0088 & 0.0024 & 0.0008 & 0.006 & 0.013 & \\
\hline $\mathrm{HcA}$ & Diff & $(1-2)$ & -0.001 & 0.003 & 0.0013 & & 0.2750 & 0.1609 \\
\hline phc & I & 14 & 0.3386 & 0.0749 & 0.02 & 0.22 & 0.48 & \\
\hline phc & $\mathrm{P}$ & 9 & 0.3789 & 0.0609 & 0.0203 & 0.29 & 0.45 & \\
\hline phc & Diff & $(1-2)$ & -0.04 & 0.0699 & 0.0299 & & 0.1915 & 0.1734 \\
\hline $\mathrm{Ah}$ & I & 14 & 0.0752 & 0.0112 & 0.003 & 0.059 & 0.092 & \\
\hline $\mathrm{Ah}$ & $\mathrm{P}$ & 9 & 0.0629 & 0.0089 & 0.003 & 0.049 & 0.075 & \\
\hline $\mathrm{Ah}$ & Diff & $(1-2)$ & 0.0123 & 0.0104 & 0.0044 & & 0.0112 & 0.0231 \\
\hline Dh & I & 14 & 0.3085 & 0.023 & 0.0061 & 0.275 & 0.342 & \\
\hline Dh & $\mathrm{P}$ & 9 & 0.2827 & 0.02 & 0.0067 & 0.251 & 0.309 & \\
\hline Dh & Diff & $(1-2)$ & 0.0258 & 0.0219 & 0.0094 & & 0.0118 & 0.0232 \\
\hline OPD & I & 14 & 10.314 & 1.234 & 0.3298 & 8.6 & 13.2 & \\
\hline OPD & $\mathrm{P}$ & 9 & 10.389 & 1.2631 & 0.421 & 8.35 & 12.05 & \\
\hline OPD & Diff & $(1-2)$ & -0.075 & 1.2452 & 0.532 & & 0.8898 & 0.8499 \\
\hline $\mathrm{AOC}$ & I & 14 & 15.979 & 4.4456 & 1.1881 & 10.79 & 24.56 & \\
\hline $\mathrm{AOC}$ & $\mathrm{P}$ & 9 & 13.28 & 3.7887 & 1.2629 & 9.44 & 21.94 & \\
\hline $\mathrm{AOC}$ & Diff & $(1-2)$ & 2.6993 & 4.2075 & 1.7976 & & 0.1481 & 0.1755 \\
\hline nOR & I & 14 & 1.2157 & 0.4418 & 0.1181 & 0.694 & 1.956 & \\
\hline nOR & $\mathrm{P}$ & 9 & 0.6882 & 0.2602 & 0.0867 & 0.158 & 1.079 & \\
\hline nOR & Diff & $(1-2)$ & 0.5275 & 0.3829 & 0.1636 & & 0.0041 & 0.0022 \\
\hline$\%$ Or & I & 14 & 91.373 & 2.9714 & 0.7941 & 83.72 & 95.31 & \\
\hline$\% \mathrm{Or}$ & $\mathrm{P}$ & 9 & 88.093 & 2.3516 & 0.7839 & 84.34 & 91.88 & \\
\hline$\%$ Or & Diff & $(1-2)$ & 3.2795 & 2.7518 & 1.1757 & & 0.0110 & 0.0074 \\
\hline
\end{tabular}

Table 5.

$\mathrm{AGE}=50+$

The TTEST Procedure

Statistics

\begin{tabular}{|c|c|c|c|c|c|c|c|c|}
\hline $\begin{array}{c}\text { Variable } \\
\text { PrWilcoxon }\end{array}$ & $\begin{array}{l}\text { Population Two- } \\
\text { Sample Test }\end{array}$ & $\mathbf{N}$ & Mean & Std Dev & Std Err & Minimum & Maximum & $\operatorname{Pr}>|\mathbf{t}|$ \\
\hline IO & I & 21 & 7.6952 & 1.1066 & 0.2415 & 5.6 & 9.8 & \\
\hline IO & $\mathrm{P}$ & 7 & 9.6429 & 1.1728 & 0.4433 & 7.9 & 11.7 & \\
\hline IO & Diff & $(1-2)$ & -1.948 & 1.1222 & 0.4898 & & 0.0005 & 0.0016 \\
\hline FR & I & 21 & 3.4952 & 0.8846 & 0.193 & 0.6 & 4.6 & \\
\hline FR & $\mathrm{P}$ & 7 & 4.0143 & 0.9856 & 0.3725 & 2.8 & 5.5 & \\
\hline FR & Diff & $(1-2)$ & -0.519 & 0.9089 & 0.3967 & & 0.2022 & 0.2742 \\
\hline $\mathrm{OA}$ & I & 21 & 0.0772 & 0.0138 & 0.003 & 0.06 & 0.11 & \\
\hline $\mathrm{OA}$ & $\mathrm{P}$ & 7 & 0.0727 & 0.0115 & 0.0044 & 0.057 & 0.09 & \\
\hline $\mathrm{OA}$ & Diff & $(1-2)$ & 0.0045 & 0.0133 & 0.0058 & & 0.4478 & 0.4096 \\
\hline po & I & 21 & 1.0476 & 0.1007 & 0.022 & 0.91 & 1.27 & \\
\hline po & $\mathrm{P}$ & 7 & 1.01 & 0.0714 & 0.027 & 0.91 & 1.14 & \\
\hline po & Diff & $(1-2)$ & 0.0376 & 0.0948 & 0.0414 & & 0.3715 & 0.3125 \\
\hline
\end{tabular}


(Table 5). Contd....

\begin{tabular}{|c|c|c|c|c|c|c|c|c|}
\hline $\begin{array}{c}\text { Variable } \\
\text { PrWilcoxon }\end{array}$ & $\begin{array}{c}\text { Population Two- } \\
\text { Sample Test }\end{array}$ & $\mathbf{N}$ & Mean & Std Dev & Std Err & Minimum & Maximum & $\operatorname{Pr}>|\mathbf{t}|$ \\
\hline $\mathrm{HcA}$ & I & 21 & 0.0085 & 0.0035 & 0.0008 & 0.004 & 0.018 & \\
\hline $\mathrm{HcA}$ & $\mathrm{P}$ & 7 & 0.0113 & 0.0067 & 0.0025 & 0.007 & 0.026 & \\
\hline $\mathrm{HcA}$ & Diff & $(1-2)$ & -0.003 & 0.0044 & 0.0019 & & 0.1567 & 0.1470 \\
\hline phc & I & 21 & 0.349 & 0.0735 & 0.016 & 0.24 & 0.53 & \\
\hline phc & $\mathrm{P}$ & 7 & 0.4043 & 0.0791 & 0.0299 & 0.33 & 0.57 & \\
\hline phc & Diff & $(1-2)$ & -0.055 & 0.0749 & 0.0327 & & 0.1029 & 0.0668 \\
\hline $\mathrm{Ah}$ & I & 21 & 0.0689 & 0.011 & 0.0024 & 0.054 & 0.094 & \\
\hline $\mathrm{Ah}$ & $\mathrm{P}$ & 7 & 0.0616 & 0.0089 & 0.0034 & 0.048 & 0.073 & \\
\hline $\mathrm{Ah}$ & Diff & $(1-2)$ & 0.0073 & 0.0106 & 0.0046 & & 0.1238 & 0.1366 \\
\hline $\mathrm{Dh}$ & I & 21 & 0.2952 & 0.0233 & 0.0051 & 0.263 & 0.346 & \\
\hline Dh & $\mathrm{P}$ & 7 & 0.2793 & 0.02 & 0.0076 & 0.248 & 0.304 & \\
\hline Dh & Diff & $(1-2)$ & 0.0159 & 0.0226 & 0.0098 & & 0.1182 & 0.1304 \\
\hline OPD & I & 21 & 11.19 & 1.6431 & 0.3586 & 7.2 & 13.4 & \\
\hline OPD & $\mathrm{P}$ & 7 & 13.657 & 1.587 & 0.5998 & 12.7 & 17.2 & \\
\hline OPD & Diff & $(1-2)$ & -2.467 & 1.6304 & 0.7115 & & 0.0018 & 0.0017 \\
\hline $\mathrm{AOC}$ & I & 21 & 17.588 & 6.0257 & 1.3149 & 8.92 & 28.66 & \\
\hline $\mathrm{AOC}$ & $\mathrm{P}$ & 7 & 25.954 & 8.7453 & 3.3054 & 15.04 & 38.59 & \\
\hline $\mathrm{AOC}$ & Diff & $(1-2)$ & -8.367 & 6.7512 & 2.9465 & & 0.0087 & 0.0196 \\
\hline nOR & I & 21 & 1.2278 & 0.5043 & 0.11 & 0.582 & 2.154 & \\
\hline nOR & $\mathrm{P}$ & 7 & 1.625 & 0.6625 & 0.2504 & 0.723 & 2.798 & \\
\hline nOR & Diff & $(1-2)$ & -0.397 & 0.5449 & 0.2378 & & 0.1068 & 0.2029 \\
\hline$\% \mathrm{Or}$ & I & 21 & 89.416 & 2.9108 & 0.6352 & 83.64 & 94.13 & \\
\hline$\% \mathrm{Or}$ & $\mathrm{P}$ & 7 & 84.991 & 6.4958 & 2.4552 & 71.24 & 90.54 & \\
\hline$\%$ Or & Diff & $(1-2)$ & 4.4243 & 4.0317 & 1.7596 & & 0.0184 & 0.0496 \\
\hline
\end{tabular}

Table 6. Summary of Sexual Dimorphism Index (After Hall [35]) for the 12 Variables Considered Per Age Class in the European Population and Easter Islanders

\begin{tabular}{|c|c|c|c|c|c|c|c|c|}
\hline & \multicolumn{2}{|c|}{ 20-29 Years } & \multicolumn{2}{|c|}{ 30-39 Years } & \multicolumn{2}{|c|}{ 40-49 Years } & \multicolumn{2}{|c|}{ 50-x Years } \\
\hline & EUROPE & EASTER I. & EUROPE & EASTER I. & EUROPE & EASTER I. & EUROPE & EASTER I. \\
\hline IO & 2.621 & - & 37.305 & - & 6.101 & -7.542 & -6.684 & -16.667 \\
\hline FR & 24.324 & - & 60.932 & - & -15.000 & -3.741 & -18.885 & 1.975 \\
\hline OA & 1.087 & - & 13.580 & - & 8.046 & 9.333 & - & -1220 \\
\hline $\mathbf{P}_{\mathbf{O}}$ & - & - & 5.607 & - & 2.679 & 99.901 & -3.883 & 0.990 \\
\hline HcA & - & - & 33.333 & - & 40.000 & 11.111 & 22.222 & -66.667 \\
\hline $\mathbf{P}_{\mathrm{Hc}}$ & - & - & 25.000 & - & 18.421 & 5.128 & 8.333 & -18.919 \\
\hline $\mathbf{A}_{\mathbf{h}}$ & - & - & 13.158 & - & 3.896 & 10.606 & -2.941 & 17.910 \\
\hline $\mathbf{D}_{\mathrm{h}}$ & 0.608 & - & 6.452 & - & 2.236 & 5.190 & -1.706 & 9.278 \\
\hline OPD & 7.764 & - & 44.207 & - & 0.387 & -6.436 & -10.261 & -11.265 \\
\hline AOC & 7.990 & - & 56.632 & - & 3.248 & 4.357 & -38.029 & 7.388 \\
\hline nOR & -1.026 & - & 99.963 & - & 5.705 & 14.270 & -42.348 & 24.408 \\
\hline$\% \mathrm{Or}$ & -0.075 & - & -2.152 & - & -3.962 & 0.340 & -2.201 & 9.081 \\
\hline
\end{tabular}




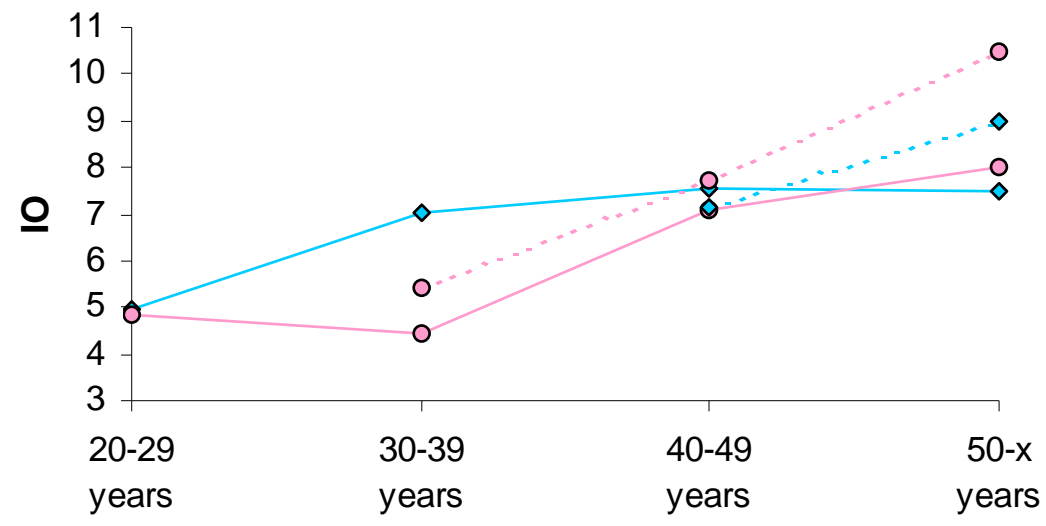

Age classes

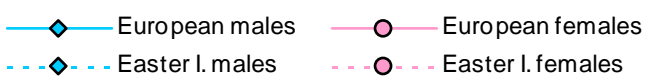

Fig. (2). Intact osteon density (IO) per age class.

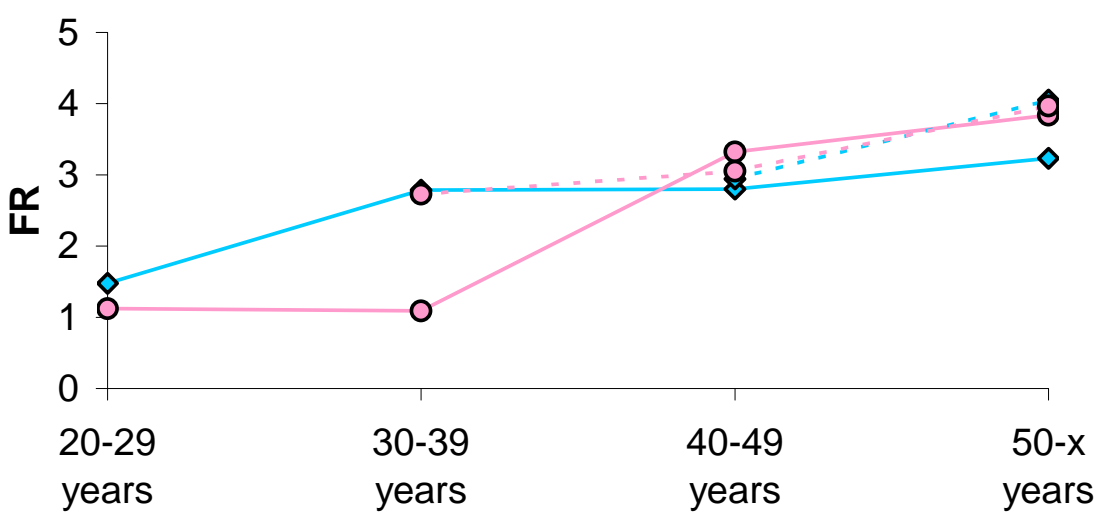

\section{Age classes}

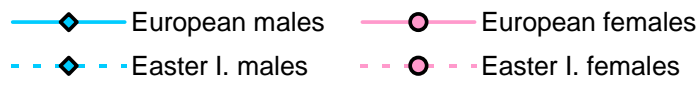

Fig. (3). Fragmentary osteon density (FR) per age class.

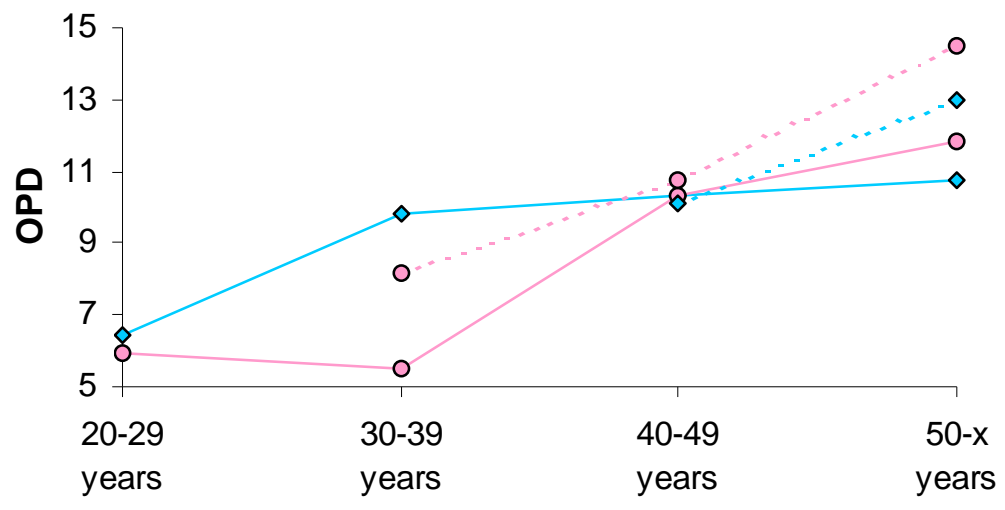

Age classes

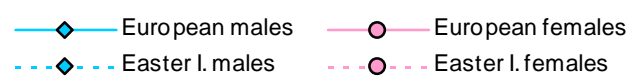

Fig. (4). Osteon population density (OPD) per age class. 


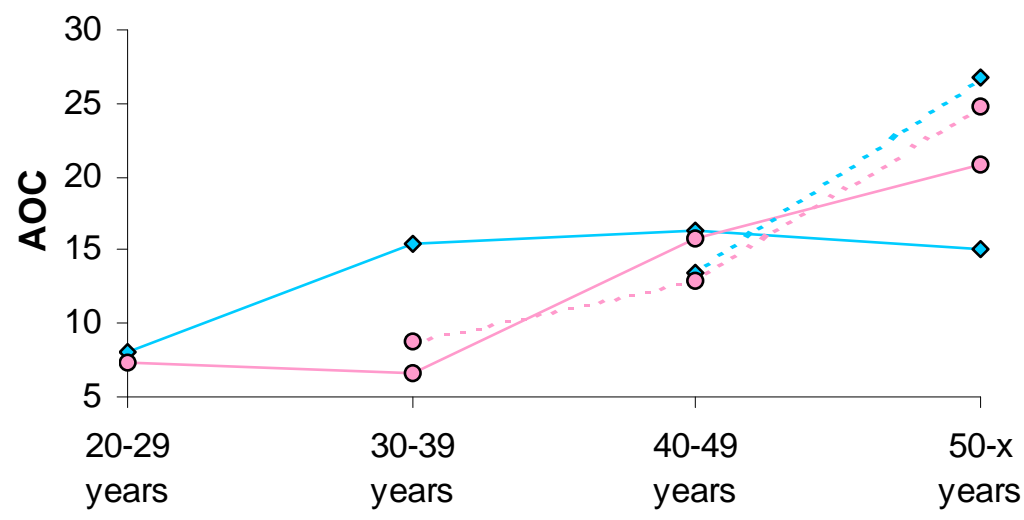

\section{Age classes}

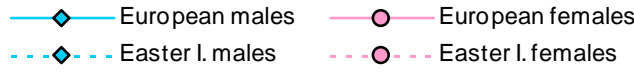

Fig. (5). Accumulated osteon creation (AOC) per age class.

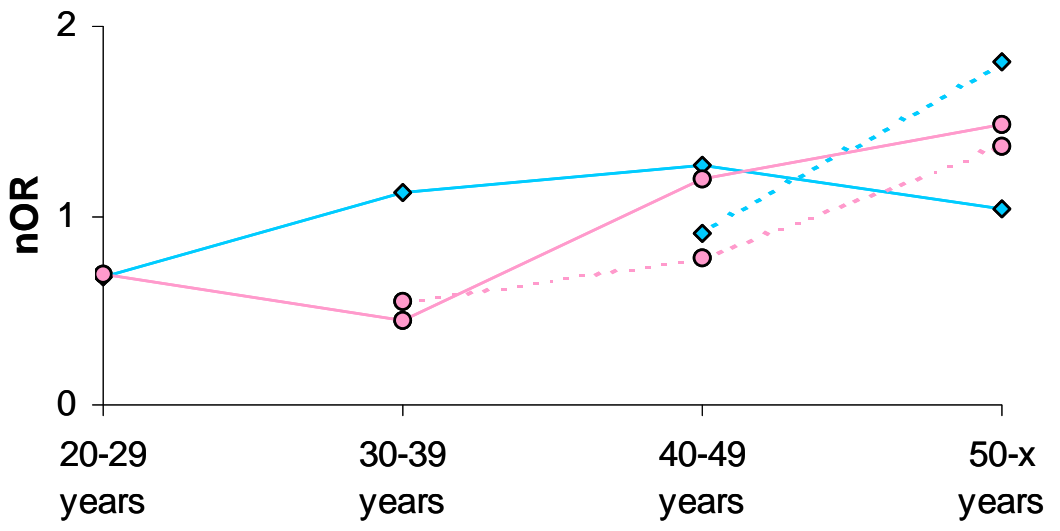

Age classes

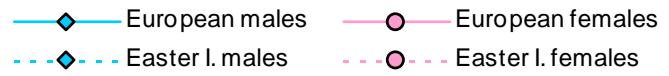

Fig. (6). Net osteonal remodeling (nOR) per age class.
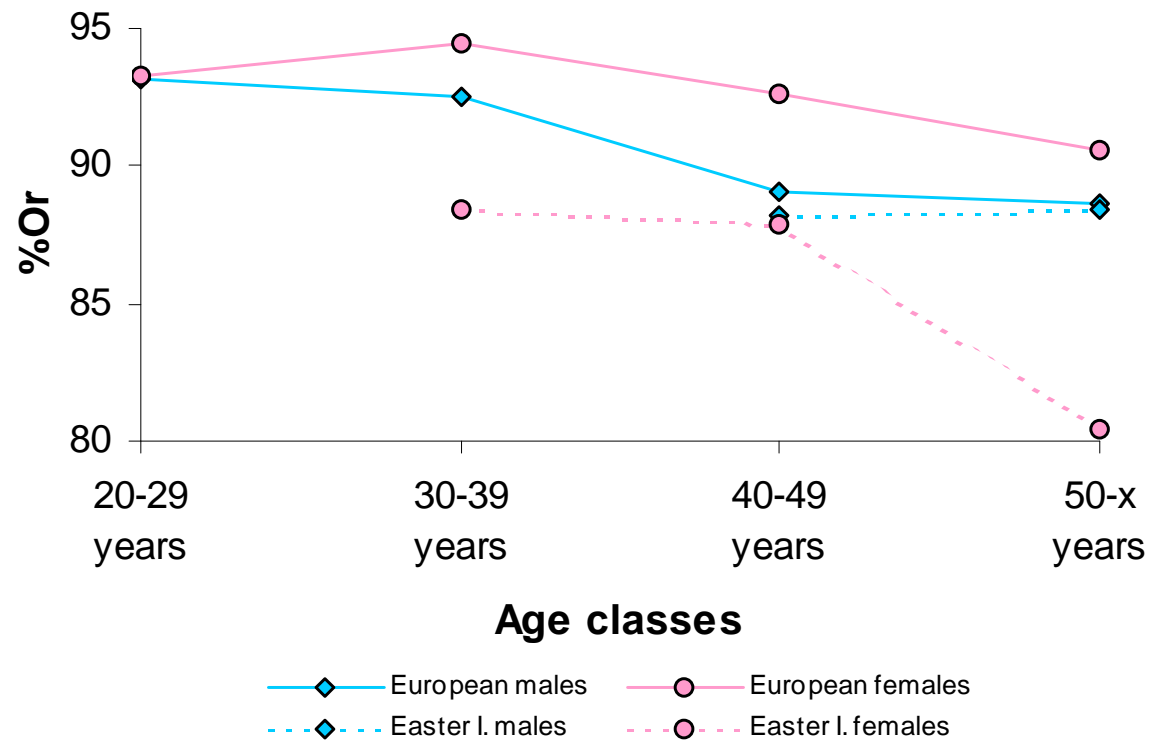

Fig. (7). Percent osteonal refilling (\%Or) per age class. 


\section{DISCUSSION AND CONCLUSION}

The histomorphometric analysis made on femoral diaphyseal sections from European and Easter Islanders populations showed the following results:

OPD increased especially in the female sample in both populations. In European males, this variable significantly increased until 30 years of age to remain constant up to death. For European females, the phenomenon was initially slower but after 40 years of age it increased rapidly, surpassing the males values. High OPD values were also registered for Easter Islanders, in both sexes but particularly for females. Macroscopic studies revealed that Polynesians humeral and femoral robusticity indices were very similar in males and females; it can be supposed that also women were submitted to intense physical stresses. Archeological evidence confirms intense building activity - especially moai building - on the island for 500 years.

- In the Easter Island female sample, OA was lower than in the European female population.

- $\quad$ Sexual dimorphism index was high for the 30 to 39 years of age-group in the European population for most variables (see Figs. 2-7).

- In the Easter Island female sample, \%Or decreased after 49 years of age. The phenomenon was also evident in both sexes of European population, although the involution process was not so marked as age advanced.

The comparative study between modern and archeological populations allows the differences in inner bone remodeling to be revealed: in this way skeletal dynamics can be linked to cultural and environmental factors. Obviously, account must be taken of the methodological errors that can derive from the intrinsic nature of the archeological material, in particular the bone's degree of conservation.

This field of study is still in its infancy, but the future should provide more archeological and paleoenvironmental information - this is the trend of current data analysis. Bone histomorphometric investigation will then be on an even stronger footing as a tool for population studies.

\section{REFERENCES}

[1] Orefici G, Drusini AG. Analysis of the cortext of burial in the plaza of Ahu Tongariki. Rapa Nui J 1997; 11(1): 29-40.

[2] Cristino FC, Vargas CP. Archaeological excavations and reconstruction of Ahu Tongariki. In: Vargas CP, Ed. Easter Island and East Polynesian Prehistory: Proceedings II International Congress on Easter Island \& East Polynesian Archaeology, Instituto de Estudios Isla de Pascua, FAU, Universidad de Chile 1999; pp. 153-8.

[3] Owsley DW, Gill GW, Ousley SD. Biological effects of OEuropean contact on Easter Island. In: Larsen CS, Milner GR, Eds. In the wake of contact. Biological responses to conquest. New York: Wiley-Liss 1994; pp. 161-77.

[4] Isçan MY, Kennedy KAR, Eds. Reconstruction of life from the skeleton. New York: Alan R. Liss, Inc 1989.

[5] Abbott S, Trinkaus E, Burr DB. Dynamic bone remodeling in later Pleistocene fossil hominids. Am J Phy Anthropol 1996; 99: 585601.

[6] Enlow DH. Remodeling of bone. Yearb Phys Anthropol 1976; 20: 19-34.
[7] Frost HM. The new bone. Some anthropological potentials. Yearb Phys Anthropol 1985; 28: 211-26.

[8] Kerley ER. The microscopic determination of age in human bone. Am J Phys Anthropol 1965; 23: 149-64.

[9] Kerley ER. Age determination of bone fragment. J Forensic Sci 1969; 14(1): 59-67.

[10] Ahlqvist J, Damsten O. A modification of Kerley's method for the microscopic determination of age in human bone. J Forensic Sci 1969; 14(2): 205-12.

[11] Dewey JR, Armelagos GJ, Bartley MH. Femoral cortical involution in three Nubian archaeological populations. Human Biol 1969; 41: 13-28.

[12] Dewey JR, Bartley MH, Armelagos GJ. Rates of femoral cortical bone loss in two Nubian populations. Clin Orthopaed Relat Res 1969; 65: 61-6.

[13] Singh IJ, Gunberg DL. Estimation of age at death in human males from quantitative histology of bone fragments. Am J Phy Anthropol 1970; 33: 373-82.

[14] Stout SD, Teitelbaum SL. Histomorphometric determination of formation rates of archeological bone. Calcif Tissue Res 1976; 21 : 163-9.

[15] Martin RD, Armelagos GJ. Morphometrics of compact bone: an example from Sudanese Nubia. Am J Phys Anthropol 1979; 51: 571-8.

[16] Martin RB, Pickett JC, Zinaich S. Studies of skeletal remodeling in aging men. Clin Orthopaed 1980; 149: 268-82.

[17] Martin RD, Magennis AL, Rose JC. Cortical bone maintenance in an historic Afro-American cemetery sample from Cedar Grove, Arkansas. Am J Phys Anthropol 1987; 74: 255-64.

[18] Thompson DD. Age changes in bone mineralization, cortical thickness, and haversian canal area. Calcif Tissue Int 1980; 31: 5-11.

[19] Stout SD. The effects of long-term immobilization on the histomorphology of human cortical bone. Calcif Tissue Int 1982; 34: $337-42$.

[20] Stout SD. Intraskeletal variation in osteon remodeling. Am J Phys Anthropol 1984; 63: 223.

[21] Stout SD. The use histomorphology to estimate age. J Forensic Sci 1988; 33(1): 121-5.

[22] Stout SD. Histomorphometric analysis of human skeletal remains. In: Isçan MY, Kennedy KAR, Eds. Reconstruction of life from the skeleton. Alan R. Liss Inc.: New York 1989a; pp. 41-51.

[23] Stout SD. The use cortical bone histology to estimate age at death. In: Isçan MY, Ed. Age markers in the human skeleton. Thomas CC, Springfield: Illinois 1989b; pp. 195-207.

[24] Simmons DJ. Options for bone aging with the microscope. Yearb Phys Anthropol 1985; 28: 249-63.

[25] Drusini AG. Refinements of two methods for the histomorphometric determination of age in human bone. Zeischrift Morphol Anthropol 1987; 77(2): 167-176.

[26] Burr DB, Ruff CB, Thompson DD. Patterns of skeletal histologic change through time: comparison of an archaic native American population with modern population. Anatomical Record 1990; 226: 307-13.

[27] Drusini AG, Businaro F. Skeletal age determination by mandibular histomophometry. Int J Phy Anthropol 1990; 5(5): 235-43.

[28] Ericksen MF. Histologic estimation of age at death using the anterior cortex of the femur. Am J Anthropol 1991; 84: 171-9.

[29] Maat GJR. Bone preservation, decay and its related conditions in ancient human bones from Kuwait. Int J Osteoarchaeol 1993; 3: 77-86.

[30] Mulhern DM, Van Gerven DP. Patterns of femoral bone remodeling dynamics in a Medieval Nubian population. Am J Phys Anthropol 1997; 104: 33-146.

[31] Pfeiffer S. Variability in osteon size in recent human populations. Am J Phys Anthropol 1998; 106: 219-227.

[32] Frost HM. Secondary osteon populations: An algorithm for determining mean bone tissue age. Yearb Phys Anthropol 1987; 30: 221-238

[33] Goodman AH, Brooke TR, Swelund AC, Armelagos GJ. Biocultural perspective on stress in prehistoric, historical and contemporary population research. Yearb Phys Anthropol 1988; 31: 169-202. 
[34] Stout SD, Paine RR. Brief communication: bone remodeling rates a test of an algorithm for estimating missing osteons. Am J Phys Anthropol 1994; 93: 123-9.
[35] Hall RL. Sexual dimorphism in Homo sapiens - A question of size. New York: Praeger 1982.

(C) Drusini et al.; Licensee Bentham Open.

This is an open access article licensed under the terms of the Creative Commons Attribution Non-Commercial License (http://creativecommons.org/licenses/by-nc/3.0/) which permits unrestricted, non-commercial use, distribution and reproduction in any medium, provided the work is properly cited. 